\title{
Electrochemical Multiplexing: Control over Surface Functionalization by Combining a Redox-sensitive Alkyne Protection Group with "Click"-Chemistry
}

Manuel Hellstern, Markus Gantenbein, Lö̈c Le Pleux, Gabriel Puebla-Hellmann, Emanuel Lörtscher, and Marcel Mayor*

In memory of Thomas Wandlowski (1958-2015)

Dr. M. Hellstern, Dr. M. Gantenbein, Dr. L. Le Pleux, Dr. G. Puebla-Hellmann, Prof. Dr. M. Mayor

Department of Chemistry, University of Basel, St. Johanns-Ring 19, CH-4056 Basel, Switzerland, E-mail: marcel.mayor@unibas.ch

Dr. G. Puebla-Hellmann, Dr. E, Lörtscher, IBM Research - Zurich, Säumerstrasse 4, CH-8803 Rüschlikon, Switzerland

Prof. Dr. M. Mayor

Institute for Nanotechnology (INT), Karlsruhe Institute of Technology (KIT), P. O. Box 3640, D-76021 Karlsruhe, Germany

Prof. Dr. M. Mayor

Lehn Institute of Functional Materials (LIFM), School of Chemistry, Sun Yat-Sen University (SYSU), Guangzhou 510275, China

Keywords: electrochemical multiplexing, redox-active protection group, surface chemistry, electrochemistry

\begin{abstract}
Local functionalization of surfaces is a current technological challenge. An electrochemically addressable alkyne protection group is presented enabling the site-selective liberation of alkynes exclusively on electrified electrodes. This controlled deprotection is based on a mendione chromophore which becomes a strong enough nucleophile upon reduction to intramolecularly attack the trialkylsilane alkyne protection group. The site-selective liberation of the alkyne is demonstrated by immobilizing the protected alkyne precursor on a transparent $\mathrm{TiO}_{2}$ electrode and subsequently immobilizing red and blue azide dyes by azide-alkyne "click" chemistry. While the proof-of-principle is based on colorations visible to the bare eye, the technique presented is generic also to non-transparent electrodes, microscale separations
\end{abstract}


and functional moieties other than dyes. It may open manifold applications where siteselective functionalization is required but hardly realizable with conventional methods.

\section{Introduction}

Immobilization of passive or functional molecular layers on surfaces is a widely applied concept ${ }^{[1-3]}$ with various applications ranging from molecular analyses, ${ }^{[4-6]}$ molecular sensing, ${ }^{[7-10]}$ and molecular electronics, ${ }^{[11,12]}$ over medical diagnostic devices ${ }^{[13-15]}$ to applications tuning macroscopic and/or environmental features like e.g. the wettability ${ }^{[16-18]}$ or the bio-compatibility ${ }^{[19-21]}$ of material surfaces. In most cases, a bi-functional molecule, combining an anchor group attaching the molecular compound to the surface with an exposed subunit representing the active moiety with tailored physio-chemical properties is used to tune the surface's terminal appearance. While such approaches are successfully applied for entire objects and surfaces of considerable dimensions and macroscopic separations, there are limitations as soon as spatial patterns of varying surface functionalities and microscopic separations are desired or a spatially constrained surface-access including buried microfluidic chips has to be overcome. For example, conventional wet chemical surface functionalization procedures are spatially limited to the size of the droplet deposited (and its subsequent surface interaction) and methods depositing the molecules from gas-phase in vacuum require advanced masking strategies. Challenging are devices with a variety of molecular features to be created in a parallel process and in a locally controlled manner, like, e.g., multidimensional sensing platforms where a multitude of nearby molecular receptors are screening analytes in parallel. Or microfluidic chips with constrained access to functional sites as the channels are locally enclosed and inaccessible for droplet deposition. Of great interest are devices analyzing molecular binding events electronically, due to their miniaturization potential and the analyte-selective binding without the requirement of labels. In such devices, each electrode is electrically addressable in a separated manner as they were contacted 
individually. Such a preexisting electrical wiring scheme is a unique feature for an immobilization strategy able to distinguish between the applied electrical potentials of each electrode and a common electrolyte. The electrochemical release of thiol-based selfassembled monolayers (SAMs) on gold electrodes has been reported. ${ }^{[22]}$ This allows the disintegration of existing SAMs. The subsequent decoration of the liberated electrode with an alternative thiol-molecule, however, is handicapped by scrambling with the remaining SAMs. Electrochemical triggered constructive build-up of molecular surfaces based on local activation or deprotection of previously assembled organic structures has been reported, ${ }^{[23]}$ e.g. based on Diels-Alder Chemistry, ${ }^{[24,25]}$ the polymerization of suitable precursors, ${ }^{[26]}$ or the release of a protection group exposing functional groups for covalent chemistry. ${ }^{[27]}$ Also electrochemical grafting of organic compounds has been reported, like the electrode functionalization by reduction of diazonium salts developed by Pinson and coworkers. ${ }^{[28]}$ Using suitable organic precursors, the technique even provided surfaces exposing alkyne groups which were subsequently further functionalized using azide-alkyne "click" chemistry (AACC) $\cdot{ }^{[29,30]}$ Numerous approaches of surface deposition experiments based on scanning probe techniques have been reported, like ,e.g., the "constructive nanolithography" from Jacob Sagiv, ${ }^{[31]}$ locally controlled electrochemistry, ${ }^{[32,33]}$ or the AFM-triggered release of an amine protection group. ${ }^{[34]}$ Probe-based functionalization, however, is not upscalable, laborintensive, time-consuming when larger areas are required and not realizable when the surface topology is complex. AACC experienced recently a tremendous development as an efficient, selective, but also mild and functional-group-tolerant coupling chemistry. ${ }^{[35,36]}$ Its integration in a novel constructive surface built-up concept would be ideal as it proved to be useful for the further functionalization of surfaces exposing alkyne groups with azide derivatives. ${ }^{[37,38]}$ While electrochemically addressable protection groups are known for a variety of functional groups, ${ }^{[39-45]}$ there are no suitable masking groups for alkynes reported so far to the best of our knowledge. We became thus interested in electrochemically-triggerable protection groups 
for alkynes as individually addressable electrodes would facilitate a site-selective molecular assembly for the reasons given above.

Here we report on the proof-of-concept for a redox-sensitive alkyne protection group that fulfills all the above-mentioned requirements of a site-selective, electrochemically triggerable protection group, compatible with AACC. Together with the deprotection concept, the synthesis of first model compounds is realized. We demonstrate further experimentally i) a first immobilization step based on anchoring groups on a metal oxide surfaces, ii) the sitespecific, local reduction on electrochemically biased electrodes, iii) the AACC-based attachment of a functional (chromophore) moiety, and iv) the sequential assembly of two functional (chromophore) moieties on patterned and differently biased electrodes. The last demonstration is referred to as electrochemical "multiplexing", a method that works only if protected compounds behave passive upon simultaneous functionalization by AACC at previously liberated, adjacent sites. All the demonstrations were visualized using anchoring groups able to bind to transparent, conductive titanium oxide $\left(\mathrm{TiO}_{2}\right)$ electrodes on which color differences are directly visible by the bare eye but can be realized on non-transparent conventional electrodes too.

\section{Results and Discussion}

The molecular design of the redox-sensitive alkyne protection group is displayed in scheme $\mathbf{1}$ and was inspired by both, the [dimethyl(oxy)propyl]dimethylsilyl (DOPS) family of alkyne protection groups developed by Vasella and co-workers, ${ }^{[46]}$ and the redox-sensitive amine protection groups reported by Carpino and co-workers. ${ }^{[47]}$ In the DOPS family, one of the three alkyl substituents of the alkynesilane comprises a masked terminal hydroxy group. After

unleashing of the hydroxy group, it acts as nucleophile and attacks the silane intramolecularly, liberating the alkine group. Caprino et al. used the increased nucleophilicity of the hydroquinone's hydroxy groups, which was attached to the masked amine via an amide 
linkage. Upon reduction of the quinone, the hydroxy-group formed attacks the amide carbonyl intramolecularly, forming a 6-membered lactone and releasing the amine group. Combining both concepts, we attach the menadione subunit as quinone precursor with an ethynyl linker to dimethylalkynesilane, resulting in a trialkylsilyl-alkine protection group displayed in (A), to which we refer to as DMEMS (DiMethyl-Ethyl(Menadionyl)Silyl) for simplicity. Upon reduction of the menadione to its hydroquinone form, the hydroxy-group attacks the silicon atom as nucleophile and the alkine substituent acts as leaving group (sketched by red arrows with the intermediate B), resulting in the liberated alkine $(\mathbf{C})$ and the 2,2-dimethyl$[1,2]$ oxasilin-6-ol derivative $\left(\mathbf{C}^{\prime}\right)$ displayed in figure $\mathbf{1}$. The selected menadione moiety as quinone structure was crucial to prevent side reactions of the highly reactive quinone system such as spontaneous dimerization, Diels-Alder cyclizations ${ }^{[48]}$ and Michael additions, ${ }^{[49]}$ while maintaining its low redox potential $\left(E_{0}=0.71 \mathrm{~V} v s\right.$. SCE in acetonitrile for menadione $) .{ }^{[50]}$ The length of the ethyl spacer is designed to result in the formation of an energetically favored 6-membered ring during the intramolecular attack. ${ }^{[47]}$

\section{(Figure 1)}

To experimentally verify the contemplated electrochemically triggered deprotection mechanism, the model compound 1 was synthesized first (synthetic protocols are available in the supporting information). As displayed in scheme 1, the synthesis started from the commercially available menadione (7), which was reduced by tin chloride under acidic conditions in methanol. The crude dihydroxy product was directly methylated using dimethyl sulfate in acetone at reflux temperature providing the dimethoxy product $\mathbf{6}$ in good yield of 68\% over both steps. ${ }^{[51]}$ Applying Rieche-formylation conditions, the dimethoxy derivative 6 was transformed to the aldehyde 5, which was isolated as white crystalline solid in $90 \%$ yield. ${ }^{[52]}$ Subsequent Wittig olefination with methyltriphenylphosphonium bromide transformed the formylated product 5 into the ethenyl decorated derivative 4 , isolated in $90 \%$ yield as colorless oil. Hydrosilylation provided the silanechloride 3 in $75 \%$ using Karstedt's 
catalyst as $\operatorname{Pt}(0)$ source. Since the silanchloride 3 is sensitive to air and moisture, the crude reaction mixture was subjected to Kugelrohr distillation after complete conversion, not only providing 3 in pure form, but also guaranteeing permanent presence of the protection gas atmosphere. A large variety of oxidation reagents and conditions were applied to the silane chloride 3 with the intention to provide an ideal protecting agent. All attempts failed and provided in the best case the cyclized 2,2-dimethyl-[1,2] oxasilin-6-ol (C' in Figure 1), pointing at the presence of either the free or even deprotonated hydroquinone species during the oxidation process. To overcome this issue, the chlorine of $\mathbf{3}$ was first substituted with the alkyne of interest, improving the compounds stability due to the considerably poorer leavinggroup features of the alkyne compared to the chloride. And indeed, treating 3 with the lithium-tert.butylphenylacetylide provided the precursor alkyne $\mathbf{2}$ as colorless oil in $86 \%$ yield after column chromatography (CC). Subsequent treatment of 2 with cerium(IV) ammonium nitrate $(\mathrm{CAN})$ in an acetonitrile/water mixture provided the bench stable DMEMS protected alkyne model compound 1 in 90\% yield as yellow oil after CC.

\section{(Scheme 1)}

With the model compound $\mathbf{1}$ at hand, the redox deprotection mechanism was first investigated in solution using sodium dithionite as reducing agent and by monitoring the reaction by GC/MS. In a dimethylsulfoxide (DMSO)/ $\mathrm{H}_{2} \mathrm{O}$ mixture (20/1), the quinone 1 was dissolved and the yellow mixture was degassed with argon for 10 minutes (black GC trace in Figure 2). An excess of the reducing agent (20 equivalents) was added at once and instantaneous decolorization of the reaction mixture was observed. The GC/MS analysis of the reaction mixture (red GC trace in Figure 2) displayed exclusively two peaks with masses corresponding to the liberated 4-tert.butylphenylacetylene and the 2,2-dimethyl-[1,2] oxasilin6-ol C'. The experiment not only shows the efficient reductive cleavage of the protection group, the observed intermediates corroborate in addition the mechanism hypothesized in

\section{figure 1.}




\section{(Figure 2)}

With the successful redox-controlled release installed, a modular approach allowing the dimethyl-ethyl(menadionyl)silyl (DMEMS) protected acetylene to be mounted on a structure comprising an anchor group moved into the focus of interest. As modular linking strategy, transition metal-catalyzed coupling chemistry was chosen and thus, the DMEMS-protected 4iodophenylacetylene $\mathbf{1 0}$ was synthesized. To avoid competing lithium iodide exchange reactions, the sterically hindered piperidinylmagnesium chloride based Knochel-Hauser base was used for the deprotonation of the acetylene to form the silyl-masked precursor 9 with the methoxy-decorated, redox-active chromophore. ${ }^{[53]}$ Treatment of the acetylene in $0^{\circ} \mathrm{C}$ cold THF with the Knochel-Hauser base and the chlorosilane 3 provided 9 as colorless oil in $91 \%$ yield after work-up and CC. Very comparable oxidation conditions as applied for $\mathbf{2}$ were applied for the transformation of $\mathbf{9}$ to $\mathbf{1 0}$, and $\mathbf{1 0}$ was isolated as yellow solid in $94 \%$ yield after CC. Sonogashira-Hagihari coupling conditions enabled the substitution of the iodine atom of $\mathbf{1 0}$ with 4-aminophenylacetylene providing the terminal amino functionalized rod $\mathbf{1 1}$ in excellent $98 \%$ isolated yield after CC. Treatment of the terminal amine with ethylenediaminetetraamide (EDTA) monoanhydride in DMF at slightly elevated temperature $\left(60^{\circ} \mathrm{C}\right)$ provided the terminally EDTA decorated molecular rod 8 in $49 \%$ yield. ${ }^{[54]}$ Purification of 8 turned out to be very challenging due to the limited solubility window of the amphiphilic compound. Best results were obtained by removing the unreacted amine by repetitively suspending 8 in acetone and decanting the solvent. However, traces of EDTA are still present in the ${ }^{1} \mathrm{H}-\mathrm{NMR}$ spectrum of the compound.

The EDTA-based anchor group was chosen due to its excellent immobilization features on transparent, conductive $\mathrm{TiO}_{2}$ electrodes,${ }^{[55-57]}$ with the latter being known for their porosity, enhancing the surface area to a level enabling direct optical observation of chemical modifications even by the bare eye. ${ }^{[58,59]}$ The commercial $\mathrm{TiO}_{2}$ electrodes consist of a glass substrates ( $3 \mathrm{~mm} \times 20 \mathrm{~mm} \times 20 \mathrm{~mm},>65 \%$ transmission) covered uniformly with a 
conductive fluorine-doped tin oxide (FTO; $\left.\mathrm{SnO}_{2}: \mathrm{F}\right)$ layer (Solaronix TCO30-8). On top, a 6 mm x $6 \mathrm{~mm}$ large area is covered with a $10-12 \mu \mathrm{m}$ thick $\mathrm{TiO}_{2}$ nanoparticle film consisting of 15-20 nm large particles. The molecular rod 8 comprising a terminal DMEMS-protected acetylene was immobilized by immersing the $\mathrm{TiO}_{2}$ electrode for 4 hours into a $3 \cdot 10^{-4} \mathrm{M}$ solution of $\mathbf{8}$ in methanol. It is noteworthy, that further, temporarily extended immersion periods did not alter the amount of immobilized molecules.

Electrochemical characteristics of the rod $\mathbf{8}$ were collected by cyclic voltammetry (CV) experiments using a three-electrode set up with a Pt disc (2 mm diameter) as working electrode, a Pt wire as counter electrode and a saturated calomel electrode (SCE) as reference. The CV displayed in Figure 3 (blue trace) was recorded with a 1mM solution of 8 in DMF with a $1 \mathrm{M}$ concentration of $\mathrm{LiCF}_{3} \mathrm{SO}_{3}$ as electrolyte. ${ }^{[60]}$ The $\mathrm{Li}^{+}$ions-containing electrolyte was chosen for comparability with the electrochemical analysis of 8 immobilized on $\mathrm{TiO}_{2}$ $\left(8 @ \mathrm{TiO}_{2}\right)$, where the flat band energy of the $\mathrm{TiO}_{2}$ surface has to be governed to enable reversibility. ${ }^{[60,61]} \mathrm{A}$ single irreversible reduction wave at about $0.68 \mathrm{~V} v$ s. SCE was observed, corresponding to the reduction of the menadione subunit to the corresponding hydroquinone form. The two-electron electrochemical process is known to proceed via the reduction to the semiquinone, which is - after immediate protonation - further reduced to the hydroquinone at a less negative potential and thus, the observation of a single-reduction wave for the twoelectron process is not surprising. ${ }^{[62,63]}$ More interesting was the irreversibility of the process with the absence of a re-oxidation wave, as this points at a complete deprotection of the reduced $\mathbf{8}$ on the time scale of the CV experiment (few seconds). Based on these promising findings, the reductive cleavage of the molecular rod immobilized on the $\mathrm{TiO}_{2}$ electrode (8@TiO2) was investigated. To keep the experimental conditions as comparable as possible to the ones described above, the functionalized $\mathrm{TiO}_{2}$ electrode was wired as working electrode together with a SCE reference electrode and platinum wire as counter in an argon-purged 0.1M LiCF $3 \mathrm{SO}_{3} /$ DMF solution. The reduction peak of the immobilized rod 8@ $@ \mathbf{T i O}_{2}$ (black 
trace in Figure 3) was at $0.79 \mathrm{~V} v s$. SCE, slightly shifted towards a more negative voltage with a considerably increased current flow due to the larger electrode surface. Also for 8@ $\mathrm{TiO}_{2}$, the irreversibility of the redox process points at a complete deprotection process during the few seconds of the CV experiment. In the subsequent CV scans (red traces in Figure 3), the reduction peak was no longer observed, suggesting that all immobilized molecules were deprotected already during the first scan. The difference of the surfaces closed by the first and the second scan (grey surface in Figure 3) corresponds to the electrons consumed to deprotect the immobilized molecules and thus allows the amount of immobilized molecules to be determined. The surface difference of $9.12 \cdot 10^{-5} \mathrm{~A} \cdot \mathrm{V}$ divided by the scan rate $(100 \mathrm{mV} / \mathrm{s})$ gives an electrical charge of $9.12 \cdot 10^{-4} \mathrm{C}$, and after division with the Faraday constant $(96485 \mathrm{C} / \mathrm{mol})$, with $9.45 \cdot 10^{-9} \mathrm{~mol}$ the amount of electrons consumed for the reduction process. As 2 electrons are required per menadione subunit, the amount of served molecules is with $4.73 \cdot 10^{-9} \mathrm{~mol}$ only half the value. By multiplication with the Avogadro number $\left(6.022 \cdot 10^{23} 1 / \mathrm{mol}\right)$ the total of addressed molecules becomes $2.85 \cdot 10^{15}$. Considering the area of the $\mathrm{TiO}_{2}$ decorated surface of $6 \mathrm{~mm}$ x $6 \mathrm{~mm}$, this gives 79 molecules 8 per $\mathrm{nm}^{2}$ of the $\mathrm{TiO}_{2}$ covered area, providing evidence for the enlargement of the active surface by the highly porous $\mathrm{TiO}_{2}$ coverage. ${ }^{[58,59]}$

To profit from the liberated alkyne in applications, it was of interest to figure out at which electrification a complete deprotection can be obtained. With a series of functionalized electrodes 8@ $\mathbf{T i O}_{2}$ it was determined that applying a potential of $-0.9 \mathrm{~V}(v s . \mathrm{SCE})$ for 10 seconds resulted in complete deprotection of the immobilized rods. It is however noteworthy, that the choice of the solvent (DMF) turned out to be crucial, as initial attempts to electrochemically deprotect decorated electrodes in acetonitrile failed.

\section{(Figure 3)}

With the electrochemically controlled liberation of the alkyne group established, the next step is the demonstration of a site-selective functionalization of wired electrode surfaces. As 
modular and widely accepted mild functionalization reaction with terminal alkynes, the copper(I)-catalyzed 1,3-dipolar Huisgen cycloaddition reaction between an azide and an alkyne group, abbreviated as AACC (azide-alkyne "click" chemistry) in the following, was the first choice. ${ }^{[35-38]}$ Employing again transparent $\mathrm{TiO}_{2}$ electrodes, the focus moved to azidedecorated dyes with the intention to detect the surface functionalization optically. To avoid unspecific adsorption of the dye structures on the polar $\mathrm{TiO}_{2}$ electrodes, the hydrophobic dyes 13 and 14 were first synthesized for that purpose. These core-substituted naphthalene diimides (NDIs) enabled a modular approach to different colors by varying exclusively the heteroatom attaching the core substituents, thus keeping important physical chemical behaviors as comparable as possible. ${ }^{[64-66]}$ 2,6-Dichloro-naphthalene-dianhydride was treated with 3azidopropan-1-amine in acetic acid at $120^{\circ} \mathrm{C}$ to result in the precursor 2,6-dichloro naphthalene diimide (NDI) 12. Due to its intrinsic poor solubility, the precursor NDI was not isolated but directly converted to the desired dyes $\mathbf{1 3}$ and $\mathbf{1 4}$. Treatment of the crude $\mathbf{1 2}$ with hexylamine and potassium carbonate $\left(\mathrm{K}_{2} \mathrm{CO}_{3}\right)$ in THF at $60^{\circ} \mathrm{C}$ for 3 days provided the blue dye 13 in 54\% yield after CC. For the red dye 14, crude 12 was treated with 1-hexylthiol in DMF at room temperature for 30 minutes. Even though the completeness of the transformation was monitored by thin layer chromatography, the red dye $\mathbf{1 4}$ was isolated in only $25 \%$ yield by $\mathrm{CC}$, due to losses in the work-up procedure.

With both dyes at hand, the immobilization sketched in scheme 2 was performed. Upon applying a potential of $-0.9 \mathrm{~V} v s$. SCE for 10 seconds in the $1 \mathrm{M} \mathrm{LiCF}_{3} \mathrm{SO}_{3} / \mathrm{DMF}$ electrolyte solution to the electrode $\mathbf{8} @ \mathbf{T i O}$, the menadione subunit was reduced to the corresponding hydroxyquinone derivative, which is unstable and decomposes within seconds to the liberated alkyne and the [1,2] oxasilin-6-ol derivative C', leaving an electrode exposing free alkynes fa@TiO2. The electrode was subsequently placed for 2 hours in a THF solution being $0.75 \mathrm{mM}$ in the dye of interest $(\mathbf{1 3}$ or $\mathbf{1 4})$, in $\mathrm{Cu}(\mathrm{MeCN})_{4} \mathrm{PF}_{6}$, and in tris(benzyltriazolylmethyl)amine (TBTA) as stabilizing ligand for the copper ions. While for the treatment with 
13, the electrodes remained intensely blue colored after extensive washing and pointing at covalently attached blue dye molecules at the electrode surface bd@TiOz, electrodes treated with 14 remained intensely red colored, as expected for red dyes immobilized on the electrode surface rd@TiO2. The transparency of the $\mathrm{FTO} / \mathrm{TiO}_{2}$ electrodes enabled the color changes to be monitored by UV-VIS spectroscopy (see supporting information Figure S-1) but also by the bare eye as displayed in scheme 2 B).

(Scheme 2)

With the coloration of the liberated alkynes established successfully, the sequential electrochemical functionalization of electrodes, entitled "electrochemical multiplexing", moved into the focus of interest. In this step, it must be proven that certain functionalized electrode areas with protected $\mathbf{8} @ \mathbf{T i O} 2$ behave passive upon deprotection and functionalizing of other electrodes areas while being exposed to the very same dye solution. As a proof-ofconcept system, the $\mathrm{TiO}_{2}$ electrode was subdivided into electrically addressable sub-areas. For that purpose, a sample, identical to the ones described above and already coated with $\mathrm{TiO}_{2}$ nanoparticles, was cut into four electrically isolated electrode quadrants. Two perpendicular cuts were made by mechanical dicing using a wafer dicing saw (ADT 71 proVectus) equipped with a $70 \mu \mathrm{m}$ thick diamond blade (Asahi H255RM). The crossing point of the two cuts was in the center of the $\mathrm{TiO}_{2}$-covered area and the entire glass substrate was cut $50 \mu \mathrm{m}$ deep to guarantee a proper electrical insulation of the buried conducting FTO layer. Thereby, the sample yields four individually addressable electrode surfaces. The $\mathrm{TiO}_{2}$-covered batch divided into four separated electrodes is displayed in Figure 4A). After immobilization of 8 on all four electrode surfaces, the pale-yellow color of the redox-active chromophore becomes clearly visible in Figure 4B). To demonstrate the sequential side-selectivity of the technique, the Deprotection-Immobilization Sequence (DIS) described above was applied only for particular subsets of electrode surfaces to immobilize the blue dye $\mathbf{1 3}$ in the presence of the still protected alkyne groups on the remaining electrodes: In Figure 4C) exclusively the upper 
right electrode was electrified, while in Figure 4E) the upper right and the lower left electrode were electrified with the conditions described above. For both steps, the other electrode surfaces (top left + bottom electrodes in Figure 4C) as well as top left and bottom right in Figure 4E)) were kept floating such that no electrochemical potential in respect to the solvent occurred and the electrochemically active deprotection group is supposed to remain on $8 @ \mathbf{T i O}_{2}$. After immersion of the electrode array in the solution enabling to immobilize the blue dye 13 by AACC, the dye is exclusively immobilized on the previously electrified electrodes, as visible by the bare eye in Figures 4C), and 4E) respectively. The electrochemical deprotection is therefore side-selective and occurs exclusively on the electrified electrode subsets of the sample. To demonstrate that the process can be repeated and that the majority of $8 @ \mathbf{T i O}_{2}$ were decorated with $\mathbf{1 3}$ by AACC in the first DIS step, the opposite electrification scheme for the electrodes was applied to deprotect the remaining 8@ $\mathrm{TiO}_{2}$ on the undecorated sites of the sample. Then, the entire electrode array was exposed again to a DIS but now using the red dye 14. As displayed in Figures 4 D) and F), the remaining electrode surfaces were colored red after electrification and exposure to the AACC solution comprising the red dye 14. The presence of the already blue-colored electrodes obviously does not interfere with the subsequent coloring of the neighboring electrodes.

(Figure 4)

\section{Conclusion}

We conclude that the combination of the redox-sensitive alkyne protection group with AACC-based functionalization is well suited for electrochemical multiplexing and yields a site-selective assembly with the required specifity. The underlaying intramolecular deprotection and click-chemistry built-up mechanisms are neither limited to transparent $\mathrm{TiO}_{2}$ electrodes nor to azide dyes as reported here. The transparent electrodes-azide dye combination chosen here, facilitates, however, unambiguous tracking of the changing surface 
terminations by color changes visible to the bare eye. Even though we demonstrated the technique to work with two azide dyes here, the technique is neither restricted to only two types of different azides nor to dye structures in general. In contrary, the novel electrochemical functionalization strategy ${ }^{[67]}$ reported here is highly generic to other electrode materials, including metals or semiconductors, such that multiplexed electrodes can be decorated subsequently with various molecular compounds exposing an azide and comprising intrinsic molecular functionalities ranging from small dyes over functional electronic or catalytic moieties to large biomolecular receptors. In fact, the steadily growing popularity of AACC further increases the diversity of available azide structures and thereby enlarges the application range of the presented unique and efficient multiplexing strategy for the siteselective molecular assembly of practical devices, including even enclosed microfluidic chips. In that respect, we are currently exploring the spatial limitations and boundary conditions of our approach by investigating the electrochemical cross-talk at ultra-dense, nanoscaled electrode arrays down to the fundamental limit of the Helmholtz layer.

\section{Supporting Information}

Synthetic protocols, analytical data, and spectra of the compounds are in the supporting information, which is available from the Wiley Online Library or from the author.

\section{Acknowledgements}

The authors acknowledge financial support by the Swiss National Science Foundation (SNF, grant number 200020-178808) and by the NCCR Molecular Systems Engineering (grant number 51NF40-141825). M.M. acknowledges support by the 111 project (90002-18011002).

Received: ((will be filled in by the editorial staff))

Revised: ((will be filled in by the editorial staff)) Published online: ((will be filled in by the editorial staff)) 


\section{References}

[1] G. M. Whitesides and P. E. Laibinis, Langmuir, 1990, 6, 87-96.

[2] S. A. Claridge, W.-S. Liao, J. C. Thomas, Y. Zhao, H. H. Cao, S. Cheunkar, A. C. Serino, A. M. Andrews and P. S. Weiss, Chem. Soc. Rev., 2013, 42, 2725-2745.

[3] A. Khassanov, H.-G. Steinrück, T. Schmaltz, A. Magerl and M. Halik, Acc. Chem. Res., 2015, 48, 1901-1908.

[4] M. Tsutsui and M. Taniguchi, Sensors, 2012, 12, 7259-7298.

[5] L. M. Goldenberg, M. R. Bryce and M. C. Petty, J. Mater. Chem., 1999, 9, 19571974.

[6] J. J. Davis, Chem. Commun., 2005, 3509-3513.

[7] J. Rickert, T. Weiss and W. Göpel, Sens. Actuators B Chem., 1996, 31, 45-50.

[8] A. Winter, S. Hoeppener, G. R. Newkome and U. S. Schubert, Adv. Mater., 2011, 23, $3484-3498$.

[9] R. M. Nyquist, A. S. Eberhardt, L. A. Silks, Z. Li, X. Yang and B. I. Swanson, Langmuir, 2000, 16, 1793-1800.

[10] K. Motesharei and D. C. Myles, J. Am. Chem. Soc., 1998, 120, 7328-7336.

[11] A. Vilan, D. Aswal and D. Cahen, Chem. Rev., 2017, 117, 4248-4286.

[12] H. B. Akkerman and B. de Boer, J. Phys. Condens. Matter, 2008, 20, 013001.

[13] B. Bottazzi, L. Fornasari, A. Frangolho, S. Giudicatti, A. Mantovani, F. Marabelli, G. Marchesini, P. Pellacani, R. Therisod and A. Valsesia, J. Biomed. Opt., 2014, 19, 017006.

[14] Y.-W. Huang, C.-S. Wu, C.-K. Chuang, S.-T. Pang, T.-M. Pan, Y.-S. Yang and F.-H. Ko, Anal. Chem., 2013, 85, 7912-7918.

[15] H. Vaisocherová, E. Brynda and J. Homola, Anal. Bioanal. Chem., 2015, 407, 39273953. 
[16] J. Osicka, M. Ilčiková, A. Popelka, J. Filip, T. Bertok, J. Tkac and P. Kasak, Langmuir, 2016, 32, 5491-5499.

[17] M. Alt, M. Jesper, J. Schinke, S. Hillebrandt, P. Reiser, T. Rödlmeier, I. Angelova, K. Deing, T. Glaser, E. Mankel, W. Jaegermann, A. Pucci, U. Lemmer, U. H. F. Bunz, W. Kowalsky, G. Hernandez-Sosa, R. Lovrincic and M. Hamburger, Adv. Funct. Mater., 2016, 26, 3172-3178.

[18] J. Casado-Montenegro, M. Mas-Torrent, F. Otón, N. Crivillers, J. Veciana and C. Rovira, Chem. Commun., 2013, 49, 8084-8086.

[19] D. J. Kim, J. M. Lee, J.-G. Park and B. G. Chung, Biotechnol. Bioeng., 2011, 108, 1194-1202.

[20] P. Ihalainen, H. Majumdar, A. Määttänen, S. Wang, R. Österbacka and J. Peltonen, Biochim. Biophys. Acta BBA - Gen. Subj., 2013, 1830, 4391-4397.

[21] M. Sezer, T. Genebra, S. Mendes, L. O. Martins and S. Todorovic, Soft Matter, 2012, $8,10314-10321$.

[22] A. Liscio, E. Orgiu, J. M. Mativetsky, V. Palermo and P. Samorì, Adv. Mater., 2010, $22,5018-5023$.

[23] J. Robertus, W. R. Browne and B. L. Feringa, Chem. Soc. Rev., 2009, 39, 354-378.

[24] M. N. Yousaf, B. T. Houseman and M. Mrksich, Angew. Chem. Int. Ed., 2001, 40, 1093-1096.

[25] W.-S. Yeo and M. Mrksich, Langmuir, 2006, 22, 10816-10820.

[26] D. Leipert, P. Heiduschka, J. Mack, H.-J. Egelhaaf, D. Oelkrug and G. Jung, Angew. Chem. Int. Ed., 1998, 37, 2337-2340.

[27] D. A. Unruh, C. Mauldin, S. J. Pastine, M. Rolandi and J. M. J. Fréchet, J. Am. Chem. Soc., 2010, 132, 6890-6891.

[28] J. Pinson and F. Podvorica, Chem. Soc. Rev., 2005, 34, 429-439. 
[29] Y. R. Leroux, H. Fei, J.-M. Noël, C. Roux and P. Hapiot, J. Am. Chem. Soc., 2010, $132,14039-14041$.

[30] Y. R. Leroux and P. Hapiot, Chem. Mater., 2013, 25, 489-495.

[31] R. Maoz, E. Frydman, S. R. Cohen and J. Sagiv, Adv. Mater., 2000, 12, 725-731.

[32] H. Liu, S. Hoeppener and U. S. Schubert, Adv. Eng. Mater., 2016, 18, 890-902.

[33] D. Bélanger and J. Pinson, Chem. Soc. Rev., 2011, 40, 3995-4048.

[34] Z. M. Fresco, I. Suez, S. A. Backer and J. M. J. Fréchet, J. Am. Chem. Soc., 2004, $126,8374-8375$.

[35] H. C. Kolb, M. G. Finn and K. B. Sharpless, Angew. Chem. Int. Ed., 2001, 40, 2004 2021.

[36] E. Haldón, M. C. Nicasio and P. J. Pérez, Org. Biomol. Chem., 2015, 13, 9528-9550.

[37] V. Castro, H. Rodríguez and F. Albericio, ACS Comb. Sci., 2016, 18, 1-14.

[38] H. He and C. Gao, Curr. Org. Chem., 2011, 15, 3667-3691.

[39] M. I. Montenegro, Electrochimica Acta, 1986, 31, 607-620.

[40] K. Arai and T. Wirth, Org. Process Res. Dev., 2014, 18, 1377-1381.

[41] C. Vaxelaire, F. Souquet, M.-I. Lannou, J. Ardisson and J. Royer, Eur. J. Org. Chem., 2009, 3138-3140.

[42] U. Ragnarsson, L. Grehn, H. L. S. Maia and L. S. Monteiro, J. Chem. Soc. Perkin 1, 2002, 97-101.

[43] M. Largeron, B. Farrell, J.-F. Rousseau, M.-B. Fleury, P. Potier and R. H. Dodd, Tetrahedron Lett., 2000, 41, 9403-9406.

[44] R. Labrecque, J. Mailhot, B. Daoust, J. M. Chapuzet and J. Lessard, Electrochimica Acta, 1997, 42, 2089-2099.

[45] A. M. Martre, G. Mousset, M. Prudhomme and E. Rodrigues-Pereira, Electrochimica Acta, 1995, 40, 1805-1809.

[46] C. Cai and A. Vasella, Helv. Chim. Acta, 1995, 78, 732-757. 
[47] L. A. Carpino, S. A. Triolo and R. A. Berglund, J. Org. Chem., 1989, 54, 33033310.

[48] C. C. Nawrat and C. J. Moody, Angew. Chem. Int. Ed., 2014, 53, 2056-2077.

[49] B. D. Mather, K. Viswanathan, K. M. Miller and T. E. Long, Prog. Polym. Sci., 2006, 31, 487-531.

[50] C. Frontana, Á. Vázquez-Mayagoitia, J. Garza, R. Vargas and I. González, J. Phys. Chem. A, 2006, 110, 9411-9419.

[51] N. Van Tuyen, B. Kesteleyn and N. De Kimpe, Tetrahedron, 2002, 58, 121-127.

[52] R. L. Nyland, M. Luo, M. R. Kelley and R. F. Borch, J. Med. Chem., 2010, 53, 1200-1210.

[53] A. Krasovskiy, V. Krasovskaya and P. Knochel, Angew. Chem. Int. Ed., 2006, 45, 2958-2961.

[54] M. Mayor, R. Scheffold and L. Walder, Helv. Chim. Acta, 1997, 80, 1183-1189.

[55] C. Mao, H. Li, F. Cui, Q. Feng and C. Ma, J. Mater. Chem., 1999, 9, 2573-2582.

[56] Q.-L. Zhang, L.-C. Du, Y.-X. Weng, L. Wang, H.-Y. Chen and J.-Q. Li, J. Phys. Chem. B, 2004, 108, 15077-15083.

[57] A. Tekiel, J. S. Prauzner-Bechcicki, S. Godlewski, J. Budzioch and M. Szymonski, J. Phys. Chem. C, 2008, 112, 12606-12609.

[58] R. N. Pandey, K. S. C. Babu and O. N. Srivastava, Prog. Surf. Sci., 1996, 52, 125192.

[59] D. Ionita, A. Mazare, C. Pirvu and I. Demetrescu, Curr. Top. Electrochem., 2010, 15, $1-15$.

[60] N. Vlachopoulos, J. Nissfolk, M. Möller, A. Briançon, D. Corr, C. Grave, N. Leyland, R. Mesmer, F. Pichot, M. Ryan, G. Boschloo and A. Hagfeldt, Electrochimica Acta, 2008, 53, 4065-4071. 
[61] P. Bonhôte, E. Gogniat, F. Campus, L. Walder and M. Grätzel, Displays, 1999, 20, $137-144$.

[62] P. D. Astudillo-Sánchez, D. Morales-Martínez, A. Sánchez, G. Rocha-Ortiz and M. Salas-Reyes, J. Electroanal. Chem., 2017, 801, 104-113.

[63] M. S. B. G. F. J. González, ARKIVOC, 2005, 172-184.

[64] M. Al Kobaisi, S. V. Bhosale, K. Latham, A. M. Raynor and S. V. Bhosale, Chem. Rev., 2016, 116, 11685-11796.

[65] S.-L. Suraru and F. Würthner, Angew. Chem. Int. Ed., 2014, 53, 7428-7448.

[66] N. Sakai, J. Mareda, E. Vauthey and S. Matile, Chem. Commun., 2010, 46, 42254237.

[67] M. Hellstern, M. Gantenbein, G. Puebla-Hellmann, M. Mayor and E. Lörtscher, US Patent Application P201701725US01, 2018. 


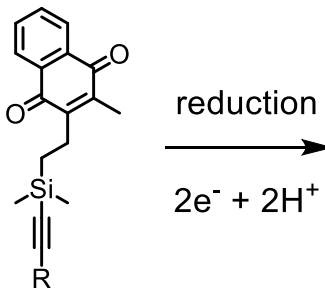

A

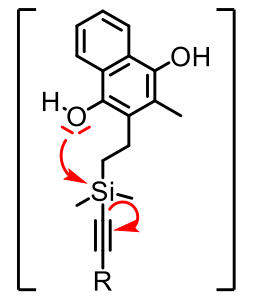

B

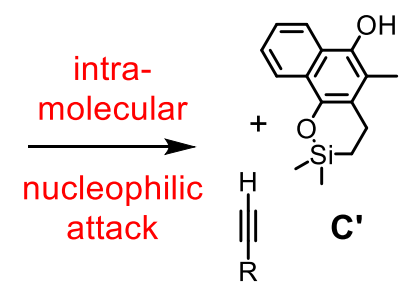

C

Figure 1. Working principle of the redox-activated alkyne protection group DMEMS. The trialkylsilyl-protection group comprises a peripheral quinone-type redox chromophore (A). Upon applying a suitable reduction potential, the redox-active chromophore is transformed into its transient hydroquinone form $(\mathbf{B})$. The decomposition mechanism of the reduced form is sketched with red arrows. The nucleophilic hydroxy groups attack spontaneously the silicon atom in an intramolecular fashion, releasing the alkyne group $(\mathbf{C})$ by forming the 2,2dimethyl-[1,2] oxasilin-6-ol derivative $\left(\mathbf{C}^{\prime}\right)$. 

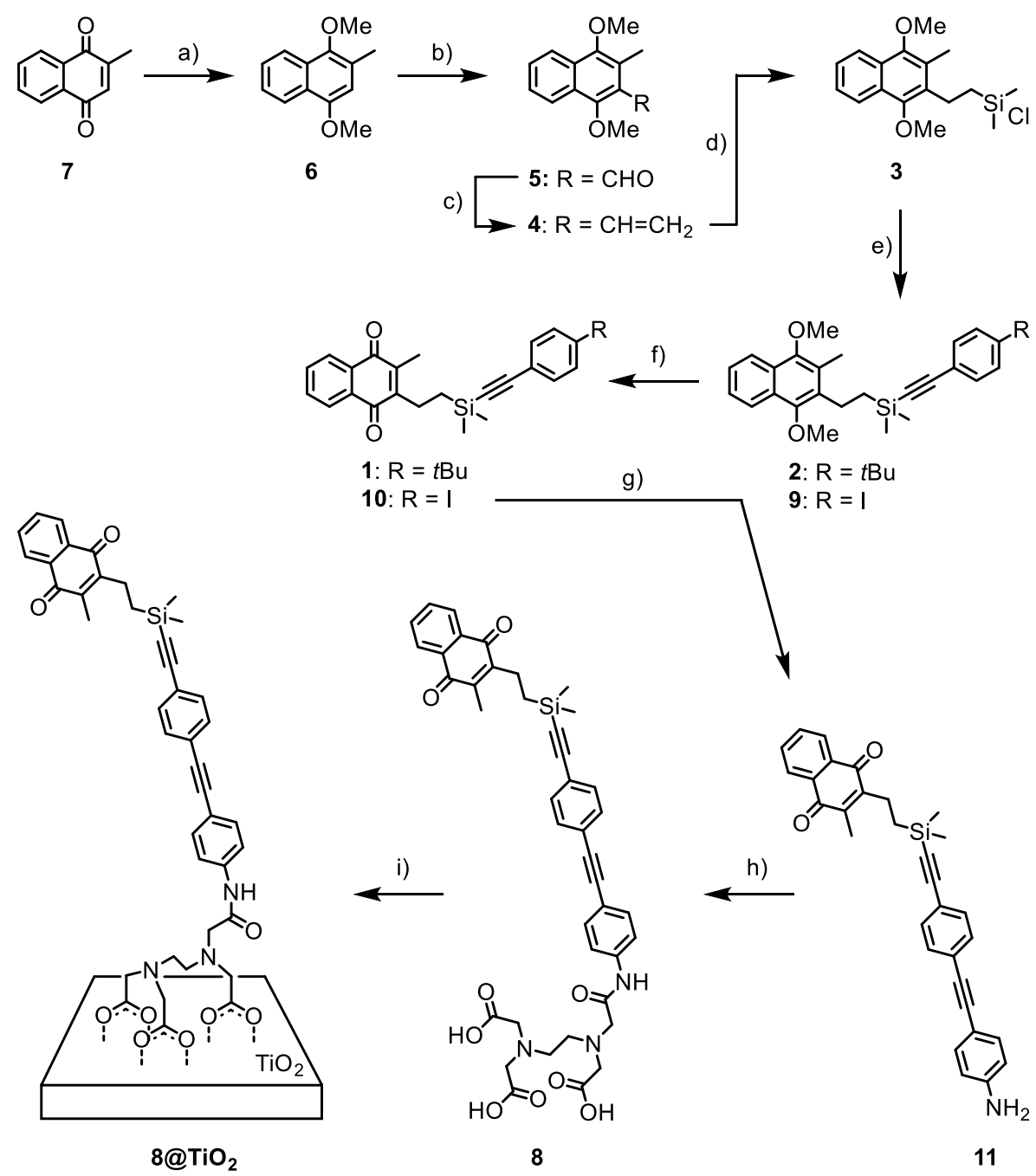

Scheme 1. Syntheses of the model compounds 1 and 8: Reagents and reaction conditions: a) $\mathrm{SnCl}_{2}, \mathrm{HCl}, \mathrm{MeOH}, 1 \mathrm{~h}$, rt; then $\mathrm{K}_{2} \mathrm{CO}_{3}, \mathrm{Me}_{2} \mathrm{SO}_{4}$, acetone, $4 \mathrm{~h}$, reflux, $68 \%$ over both steps; b) $\mathrm{TiCl}_{4}$, dichloro(methoxy)methane, $\mathrm{CH}_{2} \mathrm{Cl}_{2}, 3 \mathrm{~h}, 0^{\circ} \mathrm{C}, 90 \%$; c) $\mathrm{MeP}\left(\mathrm{Ph}_{3}\right) \mathrm{Br}, n$-BuLi, THF, $0^{\circ} \mathrm{C}$ to rt, 3h, 90\%; d) Karstedt's catalyst, dimethylchlorosilane, $34^{\circ} \mathrm{C}, 3 \mathrm{~h}, 75 \%$; e) 3 to 2: 4 tert.butylphenylacetylene, $n$-BuLi, THF, $-78^{\circ} \mathrm{C}, 1 \mathrm{~h}, 86 \% ; 3$ to 9: TMPMgCl-LiCl, 4-iodophenylacetylene, $\mathrm{THF}, 0{ }^{\circ} \mathrm{C}, 1 \mathrm{~h}, 91 \%$; f) $\mathrm{CAN}, \mathrm{MeCN} \mathrm{H}_{2} \mathrm{O}(2 / 1), \mathrm{rt}, 2$ to $1: 0.5 \mathrm{~h}, 90 \%, 9$ to 10: 1h, 94\%; g) 4-aminophenylacetylene, $\mathrm{Pd}\left(\mathrm{PPh}_{3}\right)_{4}, \mathrm{CuI}, \mathrm{Et} 3 \mathrm{~N}, \mathrm{RT}, 16 \mathrm{~h}, 98 \%$; h) EDTAmonoanhydride, DMF, $60^{\circ} \mathrm{C}, 16 \mathrm{~h}, 49 \%$; i) $\mathrm{TiO}_{2}$-electrode, $3 \cdot 10^{-4} \mathrm{M} 8$ in $\mathrm{MeOH}, 4 \mathrm{~h}, \mathrm{rt}$. 


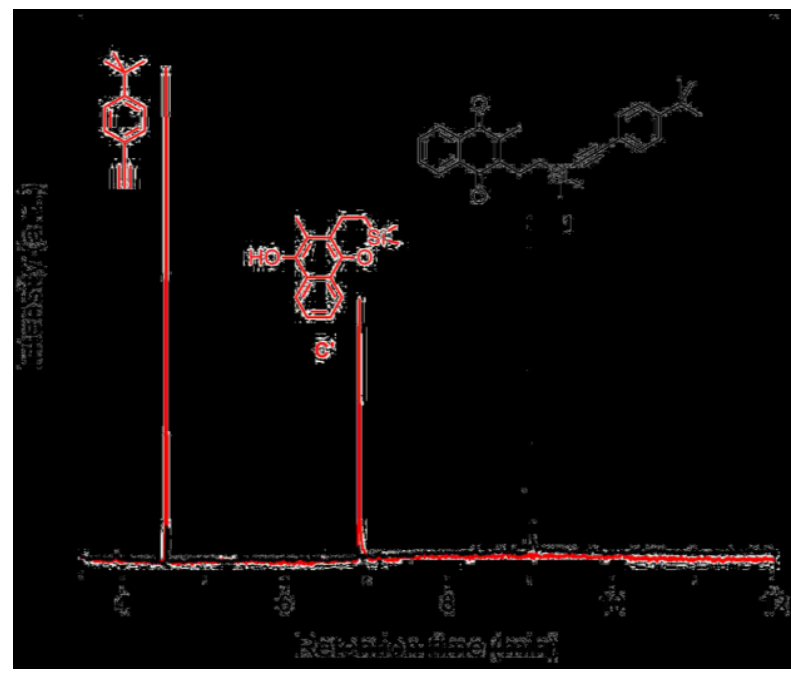

Figure 2: GC traces of the test reaction investigating the deprotection upon treatment with an excess of sodium dithionite as reducing agent. The black GC trace is the reaction mixture before addition of the reducing agent and the red GC trace the one immediately after the addition. The assignment of the peaks is based on the mass spectrometric signals of the corresponding compounds, and in the case of $\mathbf{1}$ and 4-tert.butylphenylacetylene, the retention time was further corroborated by the corresponding reference compounds.

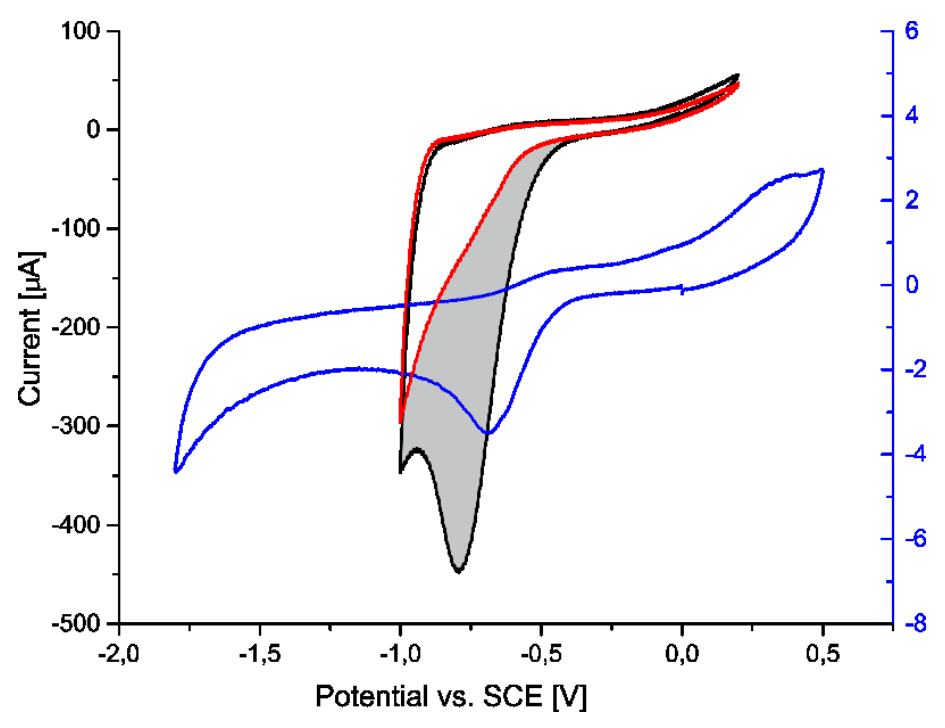

Figure 3: Cyclic voltammograms recorded in $\mathrm{DMF}$ with $1 \mathrm{M} \mathrm{LiCF} \mathrm{SO}_{3}$ as electrolyte with a scan rate of $100 \mathrm{mV} / \mathrm{s}$. The blue $\mathrm{CV}$ is the quinone 8 dissolved in the electrolyte solution. The black trace is the first cycle of the immobilized compound $\mathbf{8} @ \mathbf{T i O}_{2}$ and the red trace the 
subsequent cycle of the immobilized structure. The gray area corresponds to the amount of electrons consumed in the reduction process.

A)

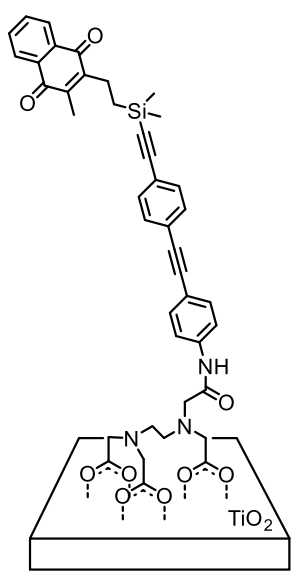

$8 @ \mathrm{TiO}_{2}$

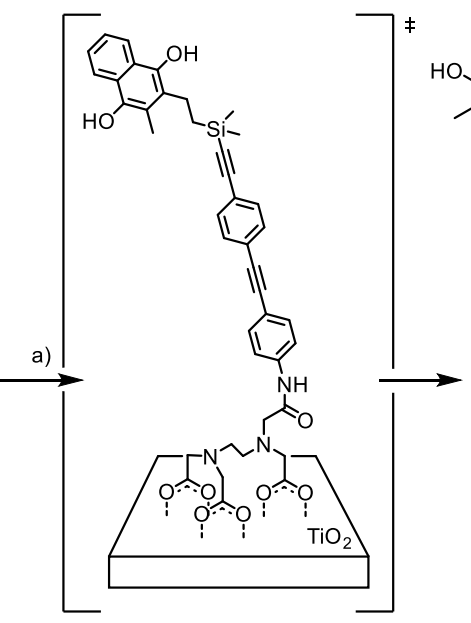

B)

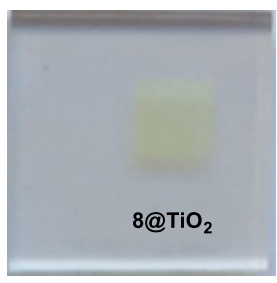

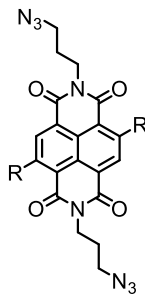

12: $\mathrm{R}=\mathrm{Cl}$

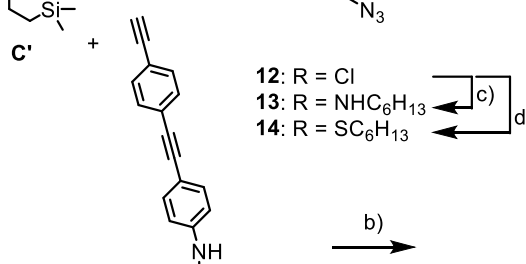

14: $\mathrm{R}=\mathrm{SC}_{6} \mathrm{H}_{13}$

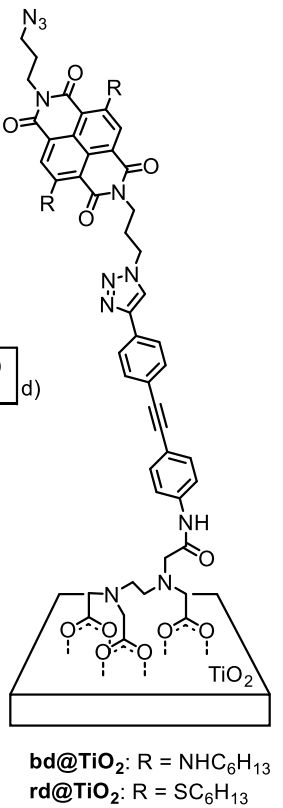

fa@TiO

Scheme 2: A) Sketch of the electrochemical deprotection and subsequent coloration by AACC sequence: Reagents and reaction conditions: a) $-0.9 \mathrm{~V} v s$. SCE for $10 \mathrm{~s}, 1 \mathrm{M} \mathrm{LiCF} 3 \mathrm{SO}_{3} / \mathrm{DMF}$, RT; b) THF, $0.75 \mathrm{~mm}$ azide dye (13 or $\mathbf{1 4}), 0.75 \mathrm{~mm} \mathrm{Cu}(\mathrm{MeCN})_{4} \mathrm{PF}_{6}, 0.75 \mathrm{~mm}$ TBTA, 2h, RT; c) crude 12, hexylamine, $\mathrm{K}_{2} \mathrm{CO}_{3}$, THF, $60^{\circ} \mathrm{C}, 3$ days, $54 \%$; d) crude 12, 1-hexanthiol, $\mathrm{K}_{2} \mathrm{CO}_{3}$, DMF, RT, 30 min, 25 \%. B) Photographs of the corresponding electrodes: 8@TiO2: pale yellow color of the redox chromophore; bd@ $@ \mathbf{T i O}_{2}$ : blue color of the immobilized blue dye 13; rd@TiO 2 : red color of the immobilized red dye $\mathbf{1 4 .}$ 

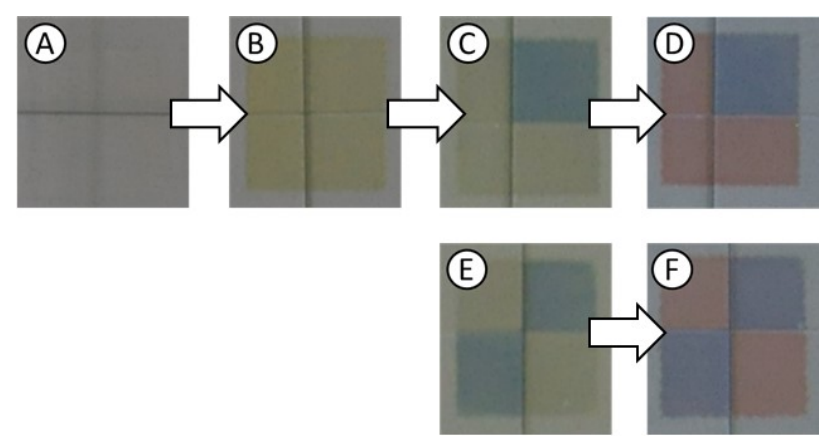

Figure 4: Photographs of the electrodes during electrochemical multiplexing: A) The parent sample with the $\mathrm{TiO}_{2}$ covered surface was cut into four individually wired electrode surfaces; B) The same sample after immobilization of $\mathbf{8}$ to yield in $\mathbf{8} @ \mathbf{T i O}_{2}$ on all four electrode surfaces; C) The same sample after applying the DIS with the blue dye to the upper right electrode resulting in bd@TiO2 exclusively for this electrode surfaces; D) The same sample after applying another DIS with the red dye giving $\mathbf{r d @ T i O _ { 2 }}$ on electrified electrodes; E) With a new sample, the upper right and lower left electrodes are first exposed upon DIS with the blue dye to give bd@TiO2, and F) shows the same sample after a DIS with the red dye to functionalize the remaining two electrodes to $\mathbf{r d} @ \mathbf{T i O}$. 


\section{Table of contents}

Electrochemically controlled functionalization of the electrode is achieved by site-selectively immobilizing red and blue dyes by azide-alkyne "click" chemistry. The alkynes exposed at the electrified electrode sections are obtained electrochemically by removing a redoxsensitive protection group.

\section{Keyword: Electrochemical Multiplexing}

M. Hellstern, M. Gantenbein, L. Le Pleux, G. Puebla-Hellmann, E. Lörtscher, M. Mayor*

Electrochemical Multiplexing: Control over Surface Functionalization by Combining a Redox-sensitive Alkyne Protection Group with "Click"-Chemistry

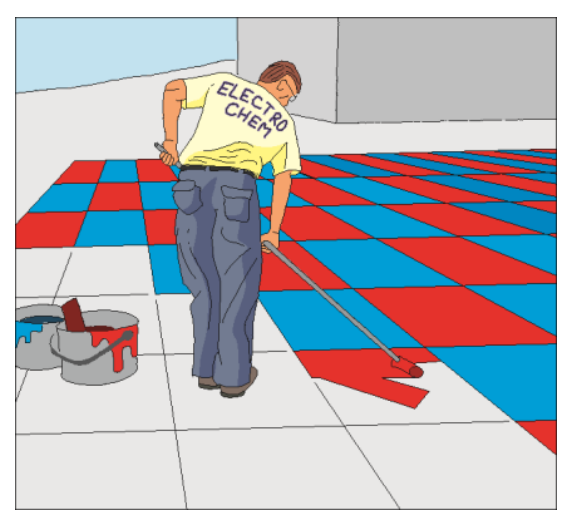

